\title{
A GENERALIZATION OF THE SCHWARZ LEMMA TO NORMAL SELFMAPS OF COMPLEX SPACES
}

\author{
JAMES E. JOSEPH and MYUNG H. KWACK
}

(Received 11 November 1998)

Communicated by P. G. Fenton

\begin{abstract}
We establish generalizations for normal selfmaps of complex spaces of the Schwarz lemma and of recent results on convergence of iterates of holomorphic selfmaps of taut spaces.
\end{abstract}

1991 Mathematics subject classification (Amer. Math. Soc.): primary 32A10, 32A17, 32H20.

Keywords and phrases: Normal, hyperbolic, tautly imbedted, fixed point.

\section{Introduction}

Let $X$ be a hyperbolic Riemann surface and $f$ a holomorphic selfmap of $X$ with a fixed point $p$. The Cartan-Carathéodory Theorem [1], a generalization of the classical Schwarz lemma, states

(i) $\left|f^{\prime}(p)\right| \leq 1$.

(ii) $f^{\prime}(p)=1$ if and only if $f=i d$ (id denotes the identity map).

(iii) $\left|f^{\prime}(p)\right|=1$ if and only if $f$ is an automorphism.

Abate [1] has shown further that

(iv) $\left|f^{\prime}(p)\right|<1$ if and only if the sequence of iterates $\left\{f^{n}\right\}$ of $f$ converges to $p$ where $f^{n}$ is defined by $f^{1}=f$ and $f^{n}=f \circ f^{n-1}$ for $n>1$.

These results have been generalized to selfmaps of taut spaces: (i), (ii) and (iii) by Wu [8] and (iv) by Abate [1] in the form of Theorem 1 (1), (2), (3), and (4) below. Theorem 1 (5) was proved by Abate [1]. The notation $\Delta$ is used for the unit disk $\{z \in \mathbb{C}:|z|<1\}$ in the complex plane $\mathbb{C}$.

(C) 2000 Australian Mathematical Society $0263-6115 / 2000 \$ A 2.00+0.00$ 
THEOREM $1.1([1,8])$. Let $f$ be a holomorphic selfmap of a taut space $X$ with a non-singular fixed point $p$. Let $d f_{p}: T_{p}(X) \rightarrow T_{p}(X)$ be the differential of $f$ at $p$. Then

(1) The eigenvalues of $d f_{p}$ have absolute values at most 1 .

(2) If $d f_{p}$ is the identity transformation of $T_{p}(X)$, then $f$ is the identity transformation of $X$.

(3) If $\left|\operatorname{det}\left(d f_{p}\right)\right|=1$, then $f$ is biholomorphic on $X$.

(4) The eigenvalues of $d f_{p}$ have absolute values less than 1 (that is $p$ is an attractive fixed point) if and only if the sequence of iterates $\left\{f^{n}\right\}$ of $f$ converges to $p$.

(5) The set of eigenvalues of $d f_{p}$ are in $\Delta \cup\{1\}$ if and only if the sequence $\left\{f^{n}\right\}$ converges and in this case the limit map is a holomorphic retraction.

Independently Kobayashi [7] and Kaup [6] (under a weaker condition) extended (1), (2) and (3) of Theorem 1 to selfmaps of hyperbolic spaces. Following the proof given by Kobayashi ([7, Theorem 5.5.1]), it can be seen from the fact that the considerations are local in nature that the proofs of (1), (2), and (3) go through virtually unchanged if the taut assumption in Theorem 1 is replaced with the requirement that the family of iterates of $f$ is a relatively compact subset of the space of continuous functions from $X$ to its one-point compactification. The proofs of (4) and (5) by Abate [1, Theorem 2.4.1, Corollary 2.4.2] also rely on this condition on the iterates which is imposed by the hypothesis placed on the space.

Let $X, Y$ be complex spaces. The notation $\mathscr{H}(X, Y)(\mathscr{C}(X, Y))$ denotes the space of holomorphic (continuous) maps from a complex space $X$ to a complex (topological) space $Y$ with the compact-open topology and $Y^{*}=Y \cup\{\infty\}$ denotes the one-point compactification of a noncompact space $Y$ and $Y^{*}=Y$ otherwise. A family of maps $\mathscr{F} \subset \mathscr{H}(X, Y)$ is uniformly normal in $\mathscr{H}(X, Y)$ if $\mathscr{F} \circ \mathscr{H}(\Delta, X)=\{f \circ g$ : $f \in \mathscr{F}, g \in \mathscr{H}(\Delta, X)\}$ is relatively compact in $\mathscr{C}\left(\Delta, Y^{*}\right)$ [4]. A map $f$ is normal when the singleton $\{f\}$ is uniformly normal. This notion of uniformly normal family extends to several variables that of uniformly normal family in one complex variable by Hayman [2] and encompasses earlier notions of normal map by various authors. Classical theorems such as the big Picard theorem and theorems for normal maps by Schottky, Pommerenke, Hahn, Järvi and Lappan have been generalized using these families [4,5]. A complex space $X$ is taut if the family $\mathscr{H}(\Delta, X) \cup\{\infty\}$ is compact in $\mathscr{C}\left(\Delta, X^{*}\right)$. A complex subspace $X$ of a complex space $Y$ is hyperbolically imbedded (tautly imbedded) in $Y$ if and only if the family $\mathscr{H}(\Delta, X)$ is relatively compact in $\mathscr{C}\left(\Delta, Y^{*}\right)(\mathscr{H}(\Delta, Y))[1,5]$ and hence holomorphic maps into $X$ are normal maps into $Y$ if $X$ is either hyperbolically imbedded or tautly imbedded in $Y$. A relatively compact hyperbolically imbedded subspace $X$ of a complex space $Y$ is obviously tautly imbedded in $Y$. 
In this paper, we establish properties $\left(1^{\circ}\right)$ and $\left(2^{\circ}\right)$ for uniformly normal families:

$\left(1^{\circ}\right)$ if $\mathscr{F} \subset \mathscr{H}(X, Y)$ is uniformly normal, then $\mathscr{F}$ is relatively compact in $\mathscr{C}\left(X, Y^{*}\right)$ (this is known to be true when $X$ is a complex manifold), and

$\left(2^{\circ}\right)$ the family of iterates of a normal selfmap $f$ of a complex space $X$ is a uniformly normal family.

As a consequence we are able to extend (1), (2), (3), (4) and the necessity part of (5) of Theorem 1 to selfmaps of complex spaces. The sufficiency part of (5) of Theorem 1 is shown to be valid for normal selfmaps where the sequence of iterates have only holomorphic subsequential limits. It follows that each normal $f \in \mathscr{H}(X, Y)$ such that $f(X) \subset X$ and such that $f(X)$ is relatively compact in $Y$ satisfies the conclusions of Theorem 1. In particular, holomorphic mappings into tautly imbedded spaces satisfy the conclusions of Theorem 1.

As another consequence we manage to unify and generalize results on holomorphic selfmaps with relative compact image of bounded domains in $\mathbb{C}^{n}$ (see [3]) and of taut domains without compact complex subspaces of positive dimension by Abate [1] in the form of the following result.

Each normal selfmap with relatively compact image of a complex space without compact complex subspaces of positive dimension has a unique fixed point and the sequence of its iterates converges to that point.

In Section 2 we present some additional preliminaries, prove $\left(1^{\circ}\right)$ and $\left(2^{\circ}\right)$ above and extend (1), (2) and (3) of Theorem 1 to normal selfmaps of complex spaces. In Section 3, we generalize to such maps (4) and the necessity part of (5) and under an additional assumption the sufficiency part of (5) of Theorem 1 on the convergence of iterates.

\section{Generalizations of Theorem 1 (1), (2), (3)}

A semi-length function, [7], on a complex space $X$ is an upper-semi-continuous non-negative function $H$ on the tangent cone, $T(X)$, such that $H(a v)=|a| H(v)$ for $a \in \mathbb{C}$ and $v, a v \in T(X)$. A length function is a semi-length function which is continuous and $H(v)>0$ for all nonzero $v \in T(X)$. We denote by $d_{H}$ the distance function generated on $X$ by $H$, that is,

$$
d_{H}(x, y)=\inf _{\gamma} \int_{a}^{b} H\left(\gamma^{\prime}(t)\right) d t
$$

where $\gamma:[a, b] \rightarrow X$ is a $C^{1}$ curve joining $x$ to $y$. The distance function $d_{H}$ is known to generate the topology on $X$ (see [7]). If $X, Y$ are complex spaces with (semi-)length 
functions $H, E$ respectively and $f \in \mathscr{H}(X, Y)$, the norm $|d f|_{H, E}$ of the tangent map for $f$ with respect to $H, E$ is defined by

$$
\begin{aligned}
|d f|_{H, E} & =\sup \left\{\left|d f_{p}\right|: p \in X\right\} \quad \text { where } \\
\left|d f_{p}\right|_{H, E} & =\sup \left\{E\left(d f_{p}(v)\right): v \in T_{p}(X), H(v)=1\right\} .
\end{aligned}
$$

We will simply use $|d f|$ and $\left|d f_{p}\right|$ when confusion is unlikely. If $X$ is a complex space, $k_{X}\left(K_{X}\right)$ will denote the Kobayashi pseudo-distance (Kobayashi-Royden semilength function [7]) on $X$. The notation $f^{*} E$ represents the pull-back of the semilength function $E$ by $f$ (that is $f^{*} E(v)=E(d f(v)$ ) for $v \in T(X)$ ).

We utilize the equivalences in Proposition 2.1 to establish $\left(1^{\circ}\right)$ above. The notation $\bar{A}$ will denote the closure of a subset $A$ of a topological space.

PROPOSITION 2.1. Let $X, Y$ be complex spaces and $H$ be a semi-length function on $X$ such that $f^{*} H \leq K_{\Delta}$ for all $f \in \mathscr{H}(\Delta, X)$. Then the following statements are equivalent for $\mathscr{F} \subset \mathscr{H}(X, Y)$.

(1) $\mathscr{F}$ is uniformly normal.

(2) $\mathscr{F} \circ \mathscr{H}(\Delta, X)$ is an evenly continuous subset of $\mathscr{H}(\Delta, Y)$.

(3) There is a length function $E$ on $Y$ such that $|d f|_{H, E} \leq 1$ for each $f \in \mathscr{F}$.

PROOF. (1) implies (2). This follows from the Ascoli-Arzelà theorem.

(2) implies (3). We begin the proof by showing that if (2) holds then for each length function $E$ on $Y$ and compact $Q \subset Y$, there exists $c>0$ such that $|d f|_{H, E} \leq c$ on $f^{-1}(Q)$ for each $f \in \mathscr{F}$. If $Q \subset Y$ is compact and fails the stated condition for the length function $E$, choose sequences $\left\{p_{n}\right\},\left\{f_{n}\right\},\left\{v_{n}\right\}$ and $q \in Q$ such that $p_{n} \in X$, $f_{n} \in \mathscr{F}, v_{n} \in T_{p_{n}}(X), f_{n}\left(p_{n}\right) \in Q, H\left(v_{n}\right)=1, f_{n}\left(p_{n}\right) \rightarrow q$ and $E\left(d f_{n}\left(v_{n}\right)\right)>n$. It follows that $E\left(d f_{n}\left(v_{n}\right)\right) \rightarrow \infty$ and that some sequence $\left\{\phi_{n}\right\}$ in $\mathscr{H}(\Delta, X)$ satisfies $\phi_{n}(0)=p_{n}$ and $\left|\left(d f_{n} \circ \phi_{n}\right)_{0}\right| \rightarrow \infty$. Let $V$ be a relatively compact neighborhood of $q$ hyperbolically imbedded in $Y$. By (2) choose $0<r<1$ such that $f_{n} \circ \phi_{n}\left(\Delta_{r}\right) \subset V$ eventually where $\Delta_{r}=\{z \in \Delta:|z|<r\}$ and consequently the set of restrictions of $f_{n} \circ \phi_{n}$ to $\Delta_{r}$ is relatively compact in $\mathscr{H}\left(\Delta_{r}, Y\right)$ contradicting $\left|\left(d f_{n} \circ \phi_{n}\right)_{0}\right| \rightarrow \infty$. To complete the proof, choose sequences $\left\{V_{n}\right\},\left\{c_{n}\right\}$ such that each $V_{n}$ is open and relatively compact in $Y, \overline{V_{n}} \subset V_{n+1}, \bigcup_{1}^{\infty} V_{n}=Y, c_{n}>0$ and $|d f|_{H, E} \leq c_{n}$ on $f^{-1}\left(\overline{V_{n}}\right)$ for each $f \in \mathscr{F}$. Choose a continuous function $\mu$ on $Y$ such that $\mu c_{n} \leq 1$ on $\overline{V_{n}}-V_{n-1}$. The function defined by $L=\mu E$ is a length function on $Y$ and $|d f|_{H, L} \leq 1$ for each $f \in \mathscr{F}$.

(3) implies (1). From (3) members of $\mathscr{F} \circ \mathscr{H}(\Delta, X)$ are distance decreasing maps with respect to the distances $k_{\Delta}$ and $d_{E}$.

PROPOSITION 2.2. Let $X, Y$ be complex spaces. If $\mathscr{F} \subset \mathscr{H}(X, Y)$ is uniformly normal, then $\mathscr{F}$ is relatively compact in $\mathscr{C}\left(X, Y^{*}\right)$. 
PROOF. Let $p \in X$ and let $U$ be a hyperbolic neighborhood of $p$. By Proposition 2.1, there are distance functions on $U$ and $Y$ with respect to which each $f \in \mathscr{F}$ is a distance decreasing map and consequently the restriction of $\mathscr{F}$ to $U$ is relatively compact in $\mathscr{C}\left(U, Y^{*}\right)$.

PROPOSITION 2.3. Let $X$ be a complex subspace of a complex space $Y$ and let $f \in \mathscr{H}(X, Y)$ be a normal map such that $f(X) \subset X$. Then the collection of iterates of $f$ is a uniformly normal subfamily of $\mathscr{H}(X, Y)$ and is relatively compact in $\mathscr{C}\left(X, Y^{*}\right)$.

PROOF. The family $\mathscr{F}$ of iterates of $f$ is uniformly normal since $\mathscr{F} \circ \mathscr{H}(\Delta, X) \subset$ $f \circ \mathscr{H}(\Delta, X)$. The relative compactness of $\mathscr{F}$ now follows from Proposition 2.2.

Let $X$ be a complex subspace of a complex space $Y$ and let $f \in \mathscr{H}(X, Y)$ satisfy $f(X) \subset X$. The notation $\mathscr{F}$ will be used for the collection of iterates of $f$ and $\mathscr{F}^{\prime}$ will denote the set of subsequential limits of $\left\{f^{n}\right\}$ in $\mathscr{C}\left(X, Y^{*}\right)$. We note that $\overline{\mathscr{F}}=\mathscr{F} \cup \mathscr{F}$ is a compact subset of $\mathscr{C}\left(X, Y^{*}\right)$ if $f$ is normal.

Theorem 2.4, a main result of the paper, generalizes (1), (2), (3) of Theorem 1 and may be viewed as a Schwarz lemma for normal selfmaps of complex spaces.

THEOREM 2.4. Let $X$ be a complex subspace of a complex space $Y$ with $p$ a nonsingular point of $X$. Let $f \in \mathscr{H}(X, Y)$ be a normal map such that $f(X) \subset X$ and $f(p)=p$ and let $d f_{p}: T_{p}(X) \rightarrow T_{p}(X)$ be the differential of $f$ at $p$. Then

(1) The eigenvalues of $d f_{p}$ have absolute values at most 1 ;

(2) If $d f_{p}$ is the identity transformation of $T_{p}(X)$, then $f$ is the identity transformation of $X$;

(3) If $\left|\operatorname{det}\left(d f_{p}\right)\right|=1$, then $f$ is biholomorphic on $X$. Corollary 2.5 derives from an observation in [5] that the identity map on $X$, id $\in \mathscr{H}(X, Y)$, is normal if and only if $X$ is hyperbolically imbedded in $Y$.

COROLlaRY 2.5. Let $X$ be a complex subspace of a complex space $Y$. Then $X$ is hyperbolically imbedded in $Y$ if and only if there is a normal map $f \in \mathscr{H}(X, Y)$ such that $f(X) \subset X$ and for a non-singular point $p \in X, f(p)=p$ and $\left|\operatorname{det}\left(d f_{p}\right)\right|=1$.

PROOF. The sufficiency follows since the identity map id $\in \mathscr{H}(X, Y)$ is normal. For the necessity we observe that it is shown in the course of the proof of Theorem 2.4 that $i d$ is a subsequential limit of the sequence of iterates of the normal map $f$ and is hence a normal map. 


\section{Generalizations of Theorem 1 (4), (5)}

We prove our next main result of the paper, Theorem 3.3, which generalizes the necessity part of Theorem 1(5) to normal selfmaps in general, and the other part to such maps under the added assumption that the sequence of iterates of $f$ contains only holomorphic subsequential limits. We will use two lemmas to prove the theorem. These lemmas, Lemma 3.1 and Lemma 3.2, are local versions of theorems for holomorphic selfmaps of taut spaces by Abate [1] and may be proved similarly. We essentially need to insure that the compositions of the maps involved are valid.

We recall that a holomorphic retraction of a complex space $X$ is a holomorphic map $\rho \in \mathscr{H}(X, X)$ such that $\rho^{2}=\rho$. It is known [1] that the image $\rho(X)$ of a holomorphic retraction $\rho \in \mathscr{H}(X, X)$, a holomorphic retract, is a closed connected complex subspace of a complex space $X$ and if $p \in \rho(X)$ is a nonsingular point of $X$, then $\rho(X)$ is also nonsingular at $p$. We will say that a map $\rho \in \mathscr{H}(U, X)$ where $U$ is open in $X$ is a holomorphic retraction on $U$ if $\rho^{2}=\rho$ on $U$. As before the image $\rho(U)$ of a holomorphic retraction $\rho$ on $U$ is a locally closed subspace of $\mathrm{X}$ (a subset $U$ of a topological space $X$ is locally closed if for each $p \in U$ there is a neighborhood $W$ of $p$ in $X$ such that $U \cap W$ is closed in $W$ ) and if $p \in \rho(U)$ is a nonsingular point of $U$, then $\rho(U)$ is also nonsingular at $p$.

LEMMA 3.1. Let $X$ be a complex subspace of a complex space $Y$. Let $f \in \mathscr{H}(X, Y)$ be a normal map such that $f(X) \subset X$. Suppose there is a nonempty open subset $U$ of $X$ such that $\alpha(U) \subset X$ for all $\alpha \in \mathscr{F}^{\prime}$. Then

(1) There exists a unique map $\rho \in \mathscr{F}^{\prime}$ such that $\rho^{2}=\rho$ on $U$ and every $\alpha \in \mathscr{F}^{\prime}$ is of the form $\alpha=\gamma \circ \rho$ on $U$ where $\gamma=\left.\alpha\right|_{U_{0}}$ is an automorphism of the complex subspace $U_{0}=\rho(U)$.

(2) $h(U)=U_{0}$ for all $h \in \mathscr{F}^{\prime}$.

(3) The restriction of $f$ to $U_{0},\left.f\right|_{U_{0}}$, is an automorphism of $U_{0}$.

PROOF. Let $\alpha \in \mathscr{F}^{\prime}$ and $f^{n_{k}} \rightarrow \alpha$. We may assume that $m_{k}=n_{k+1}-n_{k} \rightarrow \infty$, $p_{k}=m_{k}-n_{k} \rightarrow \infty, f^{m_{k}} \rightarrow \rho \in \mathscr{C}\left(X, Y^{*}\right)$ and $f^{p_{k}} \rightarrow \beta \in \mathscr{C}\left(X, Y^{*}\right)$. Since $\lim f^{m_{k}}\left(f^{n_{k}}(x)\right)=\lim f^{n_{k+1}}(x)=\alpha(x)$, we have on $U, \rho \circ \alpha=\alpha \circ \rho=\alpha$. Since $\lim f^{p_{k}}\left(f^{n_{k}}(x)\right)=\lim f^{m_{k}}(x)=\rho(x), \beta \circ \alpha=\alpha \circ \beta=\rho$ on $U$. Hence on $U$, $\rho^{2}=\beta \circ \alpha \circ \rho=\beta \circ \alpha=\rho$. From $\beta \circ \alpha=\alpha \circ \beta=\rho$ we may conclude that $\alpha$ is an automorphism of $\rho(U)=U_{0}$. Setting $\gamma=\left.\alpha\right|_{U_{0}}$, we obtain $\alpha=\gamma \circ \rho$ on $U_{0}$. The rest of the proof may proceed as in Abate's proof for the case of holomorphic selfmaps of taut spaces.

Lemma 3.2. Let $X$ be a complex subspace of a complex space $Y$ and let $f \in$ $\mathscr{H}(X, Y)$ be a normal map such that $f(X) \subset X$. If the sequence $\left\{f^{n}\right\}$ converges 
to a map $\rho \in \mathscr{C}\left(X, Y^{*}\right)$, then $\rho^{2}=\rho$ where the composition is valid and at every nonsingular point $p \in \rho(X) \cap X, d f_{p}$ has eigenvalues in $\Delta \cup\{1\}$. Conversely, if $f$ has a nonsingular fixed point $p$ such that $d f_{p}$ has eigenvalues in $\Delta \cup\{1\}$, then the sequence $\left\{f^{n}\right\}$ converges on a nonempty open subset $U$ of $X$ to a holomorphic retraction $\rho$ on $U$ and the dimension of $\rho(U)$ coincides with the multiplicity of 1 as eigenvectors of $d f_{p}$.

PROOF. If $\mathscr{F}^{\prime}=\{\rho\}$ then, from Lemma 3.1, $\rho$ is a holomorphic retraction on some neighborhood of each point $p \in \rho^{-1}(X)$ and so is holomorphic retraction on $\rho^{-1}(X)$. Hence $\rho(X) \cap X \subset \operatorname{Fix}(f)$, the set of fixed points of $f$. If $p \in \operatorname{Fix}(f)$ is nonsingular and $\lambda$ an eigenvalue of $d f_{p}$, then $\lambda^{k}$ converges to an eigenvalue of $d \rho_{p}$ which is either 0 or 1 which proves the first part of the theorem.

For the second part let $W$ be a neighborhood of $p$. We claim there is a neighborhood $U$ of $p$ so that $\alpha(U) \subset W$ for each limit $\alpha \in \mathscr{F}^{\prime}$. If not there are sequences $\left\{f^{n_{k}}\right\}$ and $\left\{z_{k}\right\}$ such that $z_{k} \rightarrow p$ and $f^{n_{k}}\left(z_{k}\right) \notin W$. But this contradicts the relative compactness of $\mathscr{F}$ in $\mathscr{C}\left(X, Y^{*}\right)$. Let $\rho \in \mathscr{F}^{\prime}$ be the limit retraction of the sequence $\left\{f^{n}\right\}$ on $U$ and $\alpha \in \mathscr{F}^{\prime}$. Now we may follow Abate's arguments and conclude from Theorem 1.4 and Lemma 3.1 that $\alpha$ is the identity map on $\rho(X) \cap U$ and hence $\alpha \circ \rho=\rho$ on $U$. Since $\alpha=\alpha \circ \rho$ on $U$, we get $\alpha=\rho$ on the connected component of $\rho^{-1}(X)$ containing $p$.

Theorem 3.3 follows from Lemma 3.2 and the fact that two holomorphic maps that agree on an open subset are identical on their common domain.

THEOREM 3.3. Let $X$ be a complex subspace of a complex space $Y$ and let $f \in$ $\mathscr{H}(X, Y)$ be a normal map such that $f(X) \subset X$. If the sequence $\left\{f^{n}\right\}$ converges, then it converges to a holomorphic map $\rho$ such that $\rho^{2}=\rho$ where the composition is valid and at every nonsingular fixed point $p$ of $f$ the differential $d f_{p}$ has eigenvalues in $\Delta \cup\{1\}$. Conversely, if $f$ has a nonsingular fixed point $p$ such that $d f_{p}$ has eigenvalues in $\Delta \cup\{1\}$ and in addition $\mathscr{F}^{\prime} \subset \mathscr{H}(X, Y)$, then $\left\{f^{n}\right\}$ converges.

The following corollary shows that (5) of Theorem 1 holds for selfmaps of tautly imbedded subspaces.

COROLLARY 3.4. Let $X$ be a tautly imbedded subspace of a complex space $Y$ and let $f \in \mathscr{H}(X, X)$. If the sequence $\left\{f^{n}\right\}$ converges in $\mathscr{H}(X, Y)$, then it converges to a holomorphic map $\rho \in \mathscr{H}(X, Y)$ such that $\rho^{2}=\rho$ whenever the composition is defined and at every nonsingular fixed point $p$ of $f$ the differential $d f_{p}$ has eigenvalues in $\Delta \cup\{1\}$. Conversely, iff has a nonsingular fixed point $p$ such that $d f_{p}$ has eigenvalues in $\Delta \cup\{1\}$ then $\left\{f^{n}\right\}$ converges. 
PROOF. We need to show that $\mathscr{F}^{\prime} \subset \mathscr{H}(X, Y)$. Suppose this is not the case and assume that $\lim f^{n_{k}}\left(z_{0}\right)=\infty \in Y^{*}$ for $z_{0} \in X$ and $n_{k}-n_{k-1} \rightarrow \infty$. Define a sequence of constant maps $\left\{\phi_{k}\right\} \subset \mathscr{H}(\Delta, X)$ by $\phi_{k}(z)=f^{n_{k+1}-n_{k}}\left(z_{0}\right)$. Since $\left\{f^{n_{k}} \circ \phi_{k}\right\}$ is relatively compact in $\mathscr{H}(X, Y)$, we get a contradiction.

Abate [1] called a nonsingular fixed point $p$ of a selfmap $f$ of a complex space $X$ an attractive fixed point if the eigenvalues of the differential $d f_{p}$ are in $\Delta$ and proved if $p$ is a nonsingular point of a taut space $X$ and $f \in \mathscr{H}(X, X)$, then $p$ is an attractive fixed point of $f$ if and only if the sequence of iterates of $f$ converges to $p$. Theorem 3.5 extends this result (Theorem 1(4)) to normal selfmaps.

THEOREM 3.5. Let $f$ be a normal selfmap of a complex space $X$ and let $p \in X$ be a nonsingular point. Then the sequence $\left\{f^{n}\right\}$ of iterates of $f$ converges to $p$ if and only if $p$ is an attractive fixed point of $f$.

Proof. The sufficiency is obvious. Suppose $p$ is an attractive fixed point of $f$. Then the limit retraction $\rho$ of the sequence $\left\{f^{n}\right\}$ defined on a neighborhood $U$ of $p$ is a constant map since the dimension of the image space $\rho(U)$ is 0 by Lemma 3.2. The proof may be finished by using the arcwise-connectedness of $X$.

In Hervé [3] it is shown that each holomorphic selfmap of a bounded domain in $\mathbb{C}^{n}$ with relative compact image has a unique fixed point. Abate [1] proved a similar result that each holomorphic selfmap with relative compact image of a taut space without compact complex subspaces of positive dimension has a unique fixed point and the sequence of its iterates converges to that point. Theorem 3.6 unifies and extends these results to normal selfmaps.

THEOREM 3.6. Let $X$ be a complex space without compact complex subspaces of positive dimension. If $f \in \mathscr{H}(X, X)$ is a normal map with relatively compact image then $f$ has an unique fixed point $p$ and its sequence of iterates converges to $p$.

PROOF. There exists a holomorphic retraction $\rho \in \mathscr{F}^{\prime}$ by Lemma 3.1. Since $\rho(X)$ is a compact subspace, the proof is completed by appeal to Lemma 3.2 .

\section{References}

[1] M. Abate, Iteration theory of holomorphic maps on taut manifolds (Mediterranean Press, Rende, Cosenza, 1989).

[2] W. K. Hayman, 'Uniformly normal families', in: Lectures on functions of a complex variable (ed. Kaplan) (Univ. of Mich. Press, 1955) pp. 199-212. 
[3] M. Hervé, Several complex variables, local theory (Oxford Univ. Press, London, 1963).

[4] J. E. Joseph and M. H. Kwack, 'Some classical theorems and families of normal maps in several complex variables', Complex Variables 29 (1966), 343-378.

[5] —. 'Extension and convergence theorems for families of normal maps in several complex variables', Proc. Amer. Math. Soc. 125 (1997), 1675-1684.

[6] W. Kaup, 'Reelle Transformationsgruppen und invariante Metriken auf komplexen Räumen', Invent. Math. 3 (1967), 43-70.

[7] S. Kobayashi, Complex hyperbolic spaces (Springer, New York, 1998).

[8] H. Wu, 'Normal families of holomorphic mappings', Acta Math. 119 (1967), 193-233.

Department of Mathematics

Howard University

Washington, DC 20059

USA

e-mail: mkwack@fac.howard.edu 